ARTIFICIAL SATELLITES, Vol. 48. No. 3 - 2013
DOI: 10.2478/arsa-2013-0011

\title{
INFLUENCE OF EPHEMERIS ERROR ON GPS SINGLE POINT POSITIONING ACCURACY
}

\author{
Lihua Ma, Meng Wang \\ National Astronomical Observatories, Chinese Academy of Sciences, Beijing 100012, China \\ e-mail:mlh@nao.cas.cn
}

\begin{abstract}
The Global Positioning System (GPS) user makes use of the navigation message transmitted from GPS satellites to achieve its location. Because the receiver uses the satellite's location in position calculations, an ephemeris error, a difference between the expected and actual orbital position of a GPS satellite, reduces user accuracy. The influence extent is decided by the precision of broadcast ephemeris from the control station upload. Simulation analysis with the Yuma almanac show that maximum positioning error exists in the case where the ephemeris error is along the line-of-sight (LOS) direction. Meanwhile, the error is dependent on the relationship between the observer and spatial constellation at some time period.
\end{abstract}

Keywords: GPS; GDOP, Single Point Positioning, ephemeris error; Yuma almanac; positioning accuracy.

\section{INTRODUCTION}

The Global Positioning System (GPS) is a space-based satellite navigation system that provides location and time information in all weather, anywhere on or near the earth, where there is an unobstructed line of sight to four or more GPS satellites. Generally, satellite geometry and an error on range measurement determine the accuracy of GPS position fix. The effect of geometry of the satellites on position error is called as geometric dilution of precision (GDOP) and it is roughly interpreted as ratio of position error to the range error (Kaplan and Hegarty 2006; Misra and Enge 2006). In many outdoor circumstances, the number of visible satellites is available to provide a small enough GDOP for positioning. The combined effects of satellite ephemeris and clock errors, propagation time delay, multipath effect and receiver measurement noise, projected on to the line-of-sight (LOS) vector from the satellite to the observer affect the range measurement. Location estimations of GPS satellites are computed and uplinked to the satellites with other navigation data message parameters for rebroadcast to the ground user. As in the case of the satellite clock corrections, these corrections are generated using a curve fit of the control segment's best prediction of each satellite's position at the time of upload. The effective pseudorange and carrier-phase errors due to ephemeris errors can be computed by projecting the satellite position error vector onto the satellite-to-user LOS vector. Along-track and cross-track components are more difficult for the control segment to observe through its monitors on the surface of the Earth, since these components do not project significantly onto LOS toward the Earth (Kaplan and Hegarty 2006). It is obvious that both magnitude and direction of ephemeris error can influence the GPS Single Point Positioning (SPP) accuracy. In this study, a direction 
influence on SPP accuracy is researched. An ephemeris error sphere surface and error vector is presented in the Section 4. Simulation analysis is given in the Section 3 and a Summary is presented in the final Section.

\section{EPHEMERIS ERROR}

The gravitational and non-gravitational forces perturb the motion of the GPS satellites, causing the orbits to deviate from a Keplerian ellipse in the inertial space (George and Collins 2004). The perturbations are characterized by long-term, periodic and anomaly components, and must be continually determined through the analysis of tracking data. To adequately describe the orbit of GPS satellites during the interval of time for which the ephemeris information is transmitted. A representation based on Keplerian elements plus perturbations is used. Estimates of ephemerides for all satellites are computed and uplinked to the satellites with other navigation data message parameters for rebroadcast to the user. Generally the satellite ephemerides are broadcast several hours in advance of the epoch for which their locations are calculated (Kaplan and Hegarty 2006; Misra and Enge 2006). Although the Keplerian representation has physical meaning, additional parameters are required to model the perturbations about the satellite orbit. These parameters are obtained from a curve fit to the predicted satellite ephemeris. They are not true Keplerian elements as they only describe the ephemeris over the interval of applicability and not for the whole orbit.

Considering that the satellite ephemeris has errors in all three dimensions, therefore a part of the error will appear as a common range error and the rest will remain as a residual ephemeris error with typical magnitudes in the range of 1-6 m (Warren and Raquet 2002). The position error in broadcast ephemeris is a vector that is depicted in Fig. 1.

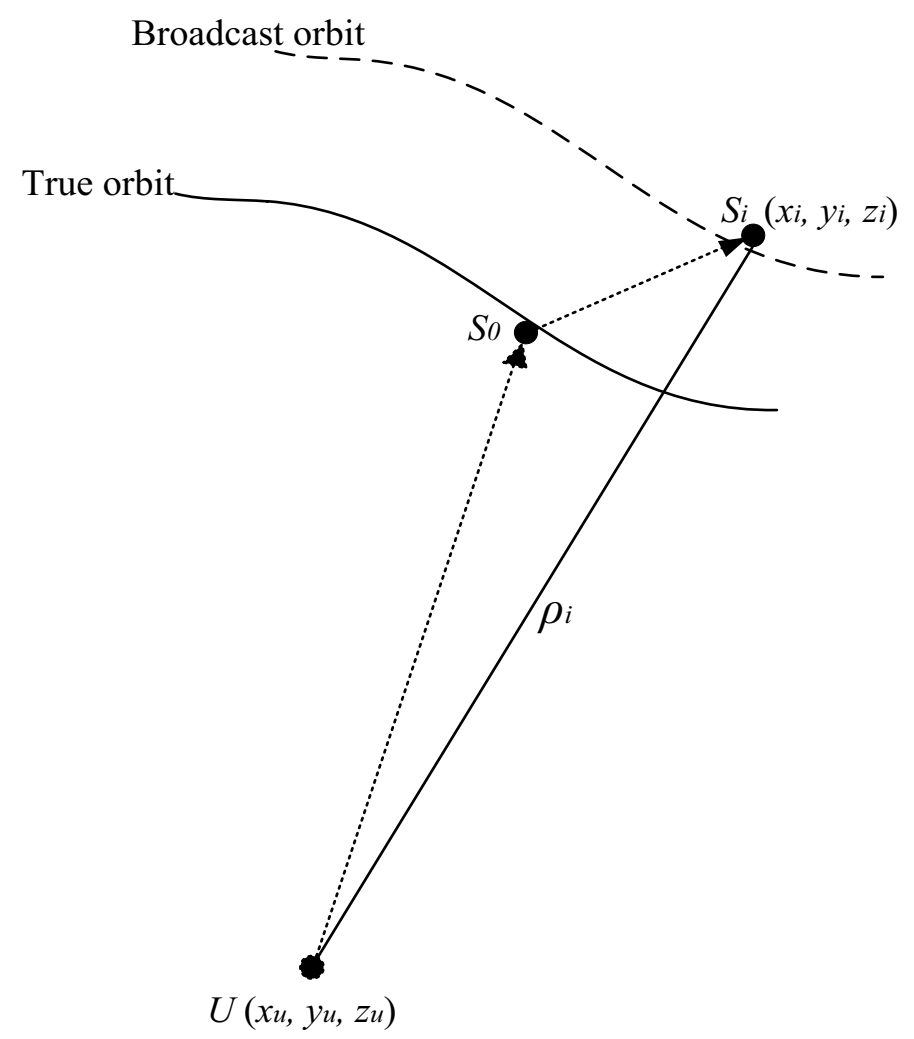

Fig. 1. Pseudorange measurement schematic diagram 
To determine the user 3D-location $\left(x_{u}, y_{u}, z_{u}\right)$ and $t_{u}$ is a combination of signal reception time, signal broadcast time, error of receiver clock and error of satellite clock. The pseudorange observation equation is as follows:

$$
\rho_{i}=\left\|S_{i}-U\right\|+c t_{u}
$$

where $\rho_{i}$ is pseudorange from the $i$ th satellite to the receiver. Equation 1 can be expanded into the following equation with the unknowns $x_{u}, y_{u}, z_{u}$ and $t_{u}$ :

$$
\rho_{i}=\sqrt{\left(x_{i}-x_{u}\right)^{2}+\left(y_{i}-y_{u}\right)^{2}+\left(z_{i}-z_{u}\right)^{2}}+c t_{u}
$$

The satellite is located at coordinates $\left(x_{s}, y_{s}, z_{s}\right)$ in the Earth-Centered Earth-Fixed (ECEF) Cartesian coordinate frame.

\section{POSITION ERROR}

The solution is given in Equation (2) using least square estimates (Langley, 1999; Kaplan and Hegarty 2006; Misra and Enge 2006):

$$
\Delta x=-\left(A^{T} A\right)^{-1} A^{T} \Delta \rho
$$

where $\Delta \mathrm{x}$ is the four-element vector of 3D-location $\left(x_{u}, y_{u}, z_{u}\right)$ and $t_{u} ; A$ denotes a matrix with each row composed of three elements of direction cosine vector, and an entry of 1 in the last column; $\Delta \rho$ is the vector of differences between the corrected pseudorange measurements and modeled pseudorange values based on the linearization point coordinates. Assuming that the measurement errors are random and the same for all observations with a common standard deviation $\left(\sigma_{U E R E}\right)$, then the covariance matrix of the parameter estimates $C_{\triangle x}$ can be described as:

$$
C_{\Delta x}=\left(A^{T} A\right)^{-1} \cdot \sigma_{U E R E}^{2}=H \cdot \sigma_{U E R E}^{2}
$$

Where $H=\left(A^{T} A\right)^{-1}$. The diagonal elements of $C_{\Delta x}$ are the variances of estimated receiver coordinates and clock offset. The geometric DOP parameters are defined as follow:s

$$
\begin{aligned}
& \text { Geometric DOP }(\text { GDOP })=\sqrt{H_{11}+H_{22}+H_{33}+H_{44}} \\
& \text { Position DOP }(\mathrm{PDOP})=\sqrt{H_{11}+H_{22}+H_{33}} \\
& \text { Horizontal DOP }(\mathrm{HDOP})=\sqrt{H_{11}+H_{22}} \\
& \text { Vertical DOP }(\mathrm{VDOP})=\sqrt{H_{33}} \\
& \text { Time DOP }(\mathrm{TDOP})=\sqrt{H_{44}}
\end{aligned}
$$

The DOP parameters provide statistically characterization of the user-satellite geometry. The quality of the estimates can be described as: 


$$
\sigma_{j}=(D O P)_{j} \cdot \sigma_{U E R E}
$$

Where $(D O P)_{j}$ is corresponding to GDOP, PDOP, HDOP, VDOP or TDOP, and $\sigma_{j}$ designates the variance of corresponding estimation error.

\section{SIMULATION ANALYSIS}

\subsection{Ephemeris error sphere}

Given the maximum ephemeris error is $r_{e}$, the satellite locates sphere surface whose center is at the true satellite location $S_{0}$ with the radius $r_{e}$. The spatial vector relationship is given in the following Fig. 2.

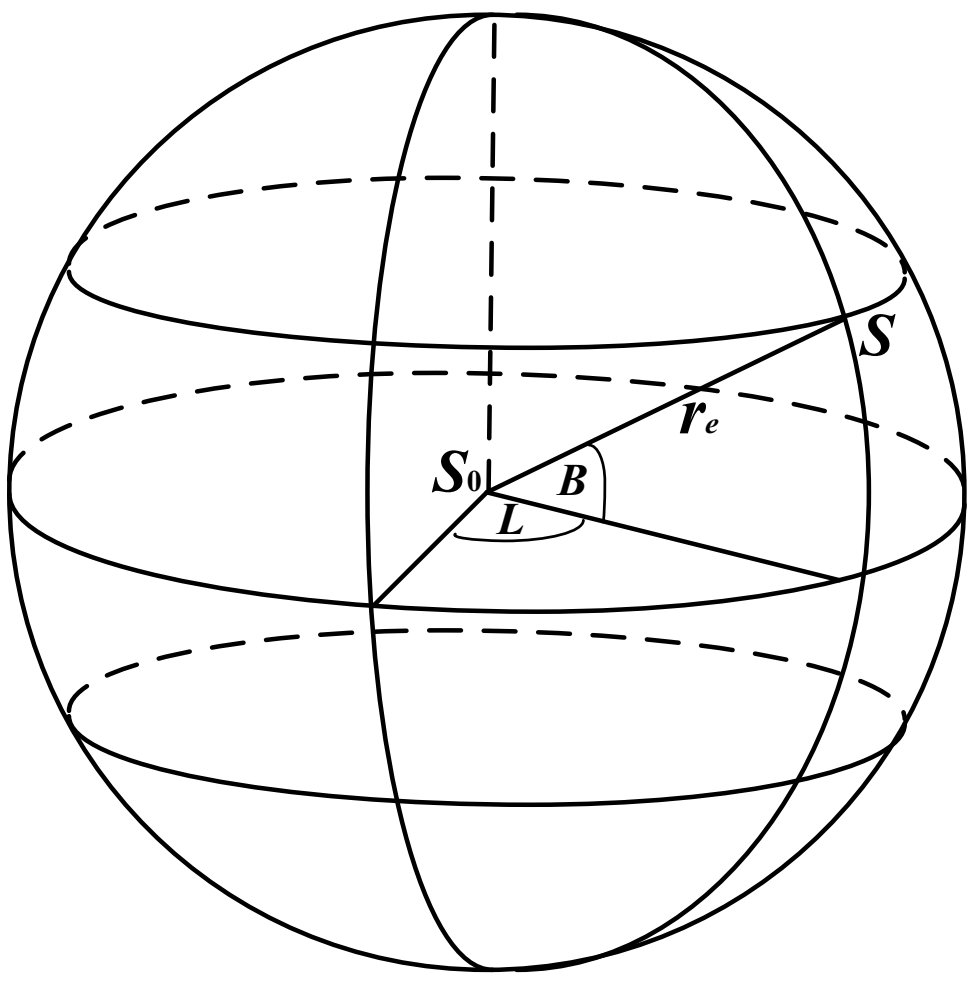

Fig. 2. Ephemeris error sphere.

Establishing a spatial Cartesian coordinate system to describe the place of broadcast satellite $S$ in the ephemeris error sphere, here the $S_{0}$ is taken as the origin of coordinates, three axes are parallel to the $x, y$ and $z$ axes with the ECEF system, respectively. Furthermore similar to the longitude of the Earth, $L$ is the angle the with the $x$-axis direction angle in the $x y$ plane; $B$ is the angle of $S_{0}$ to $S$ in the $x z$ plane. The coordinates of point $S$ are as follows:

$$
\left(\begin{array}{l}
x \\
y \\
z
\end{array}\right)=\left(\begin{array}{l}
r_{e} \cos B \cos L \\
r_{e} \cos B \sin L \\
r_{e} \sin B
\end{array}\right)
$$

With the Equation (7), the coordinate value of any point on the sphere can be obtained. 


\subsection{GPS Yuma almanac}

To investigate the ephemeris error influence on GPS SPP, we constitute a constellation with YUMA almanac. The YUMA almanac is an ASCII message containing the almanac information in YUMA format. The almanac includes only ephemeris and clock data, and does not include covariance, nor the high accuracy GPS to UTC time correlation.

The simulation is setup for May 4, 2012 and the corresponding GPS constellation is based on a YUMA almanac for GPS week 662 and reference epoch $t_{o e}$ is 503808 second.

\subsection{Analysis results}

Here supposing the maximum value of ephemeris error is 5 meters. Let $L, B$ change from $-180^{\circ}$ to $180^{\circ}$, from $-90^{\circ}$ to $90^{\circ}$ with the $5^{\circ}$ interval, respectively, similar to the geographic grid. Every grid point is corresponding to ephemeris error vector. Considering the different performance with different user location, here we choose 8 the International GNSS Service (IGS) stations at Beijing, Ulan Bator, Tokyo, New Delhi, Singapore, Jakarta, Canberra, Zhongshan stations, respectively. The position of these stations in geodetic coordinate is listed in the Table 1.

Table 1. Geodetic coordinates of observation stations.

\begin{tabular}{ccccc}
\hline & Beijing & Ulan Bator & Tokyo & New Delhi \\
\hline Latitude $(\varphi)$ & $39^{\circ} 54^{\prime} 50^{\prime \prime} \mathrm{N}$ & $47^{\circ} 55^{\prime} 0^{\prime \prime} \mathrm{N}$ & $35^{\circ} 42^{\prime} 2^{\prime \prime} \mathrm{N}$ & $28^{\circ} 36^{\prime} 50^{\prime \prime} \mathrm{N}$ \\
Longitude $(\boldsymbol{\lambda})$ & $116^{\circ} 23^{\prime} 30^{\prime \prime} \mathrm{E}$ & $106^{\circ} 55^{\prime} 0^{\prime \prime} \mathrm{E}$ & $139^{\circ} 42^{\prime} 54^{\prime \prime} \mathrm{E}$ & $77^{\circ} 12^{\prime} 32^{\prime \prime} \mathrm{E}$ \\
Altitude $(\boldsymbol{h})$ & $44 \mathrm{~m}$ & $1350 \mathrm{~m}$ & $17 \mathrm{~m}$ & $216 \mathrm{~m}$ \\
\hline Table 1. (cont.) & & & & \\
\hline \multicolumn{7}{c}{ Singapore } & Jakarta & Canberra & Zhongshan \\
\hline Latitude $(\boldsymbol{\varphi})$ & $1^{\circ} 17^{\prime} 0^{\prime \prime} \mathrm{S}$ & $6^{\circ} 12^{\prime} 0^{\prime \prime} \mathrm{S}$ & $35^{\circ} 18^{\prime} 29^{\prime \prime} \mathrm{S}$ & $69^{\circ} 22^{\prime} 44^{\prime \prime} \mathrm{S}$ \\
Longitude $(\boldsymbol{\lambda})$ & $103^{\circ} 50^{\prime} 0^{\prime \prime} \mathrm{E}$ & $106^{\circ} 48^{\prime} 0^{\prime \prime} \mathrm{E}$ & $149^{\circ} 7^{\prime} 28^{\prime \prime} \mathrm{E}$ & $76^{\circ} 22^{\prime} 40^{\prime \prime} \mathrm{E}$ \\
Altitude $(\boldsymbol{h})$ & $164 \mathrm{~m}$ & $7 \mathrm{~m}$ & $580 \mathrm{~m}$ & $11 \mathrm{~m}$ \\
\hline
\end{tabular}

At every fixed location, the mask angle of the user is 10 degrees. Based on the principle of the minimum GDOP, the best four satellites can be chosen and used to calculate location and time offset.

Here the maximum ephemeris error is only added to some satellite, at every ephemeris error vector on the surface of error sphere, single point positioning solution is obtained. Positioning error is divided into three components including east, north and up directions. Meanwhile the angle between LOS vector and error vector is calculated, the angle with $0^{\circ}$ and $180^{\circ}$ is corresponding to error vector along LOS direction. Error vector angle and positioning error distributions at above stations are given in the following Figs. 3-10. 
(a) Angles between LOS and $r_{e}$ (deg)

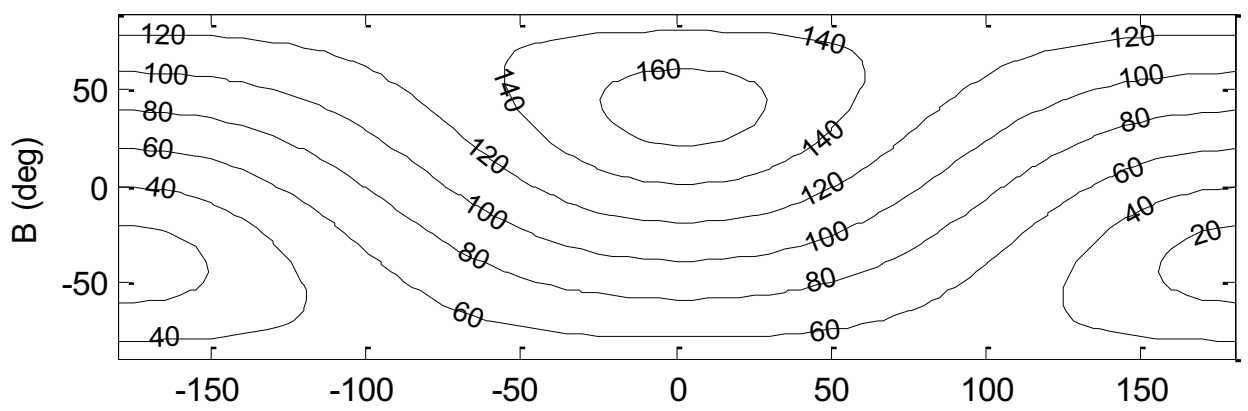

(b) East error (m)

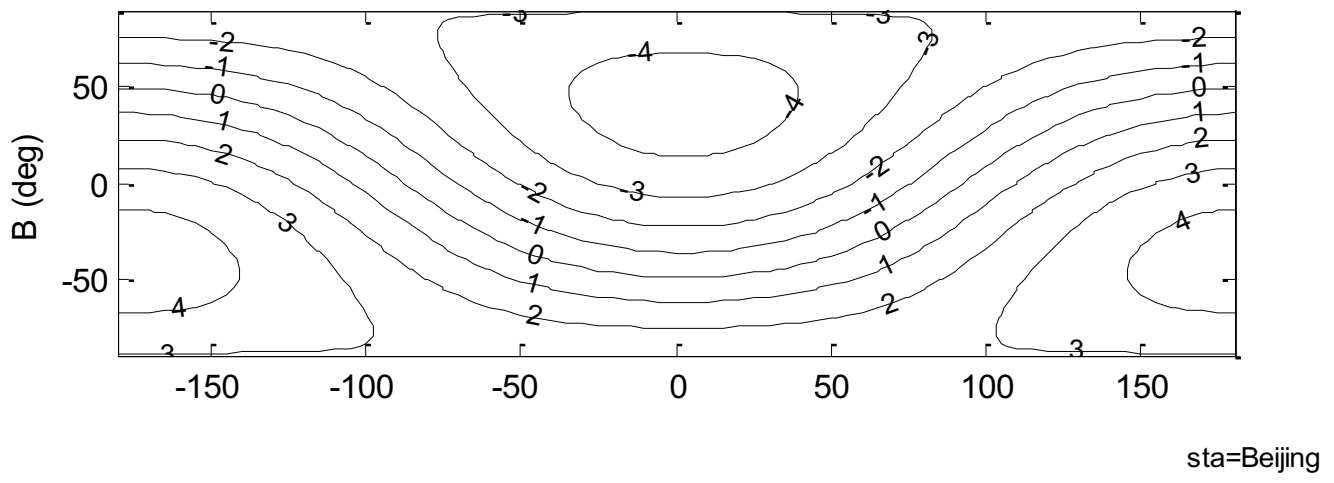

(c) North error (m)

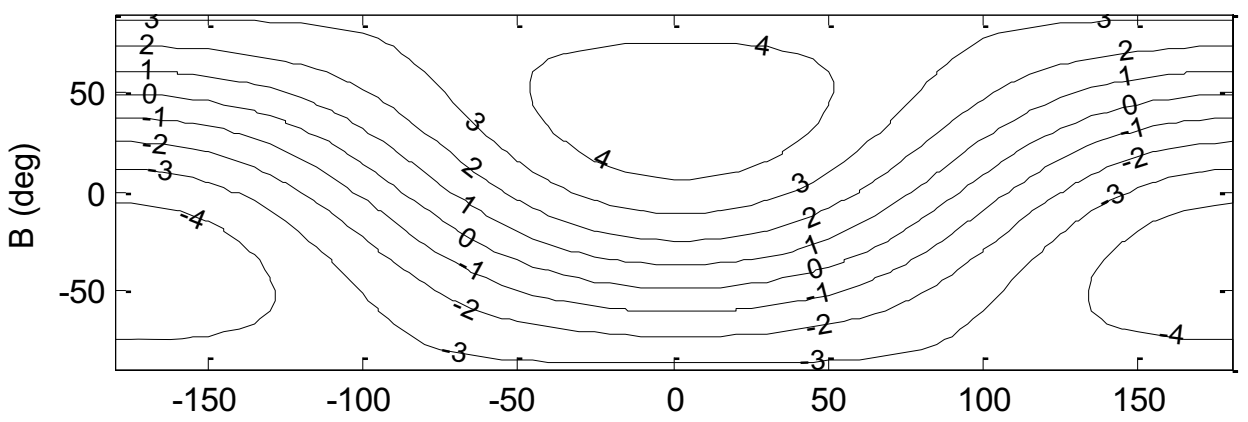

(d) Up error (m)

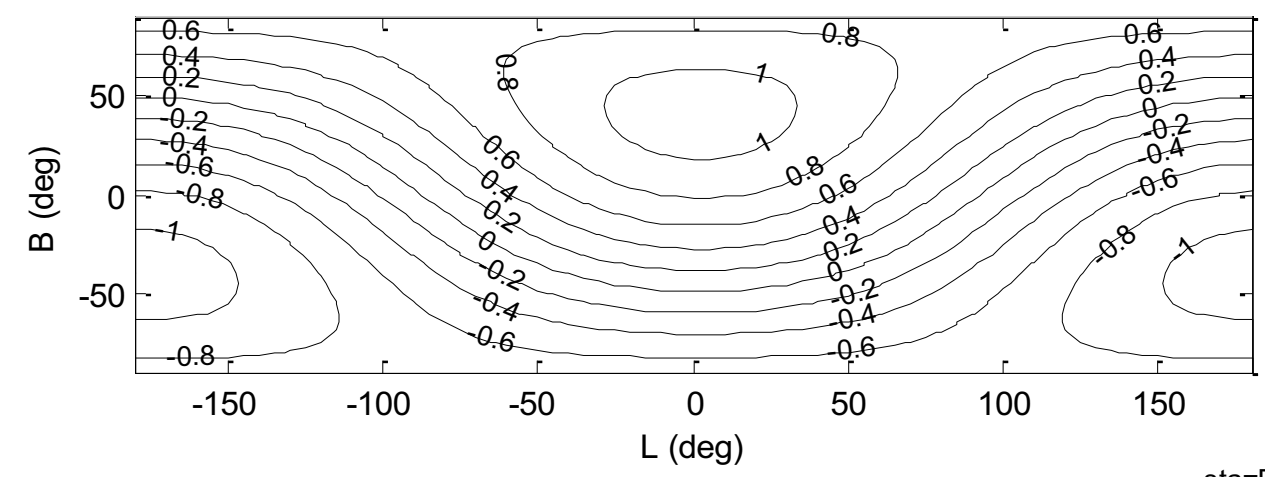

Fig. 3 Error vector angle and positioning error distributions at Beijing 
(a) Angles between LOS and $r_{e}$ (deg)

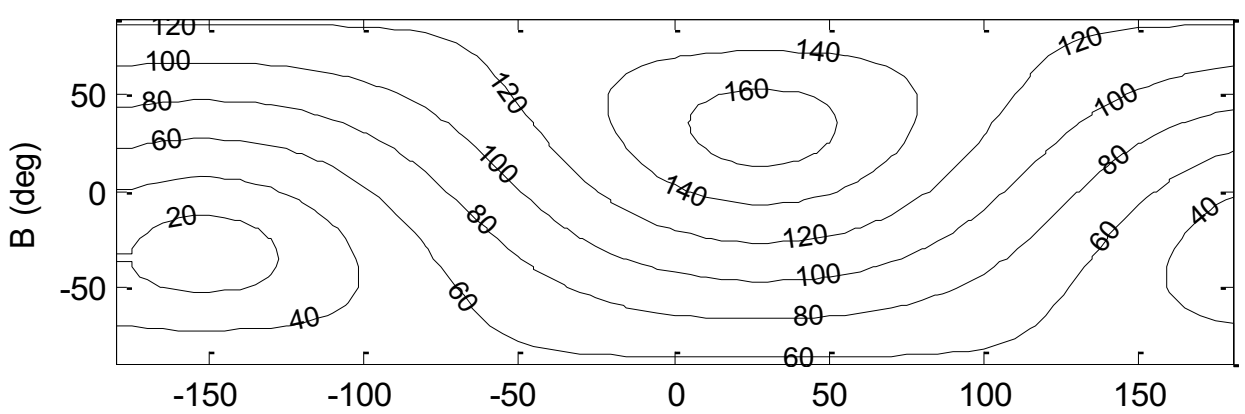

(b) East error (m)

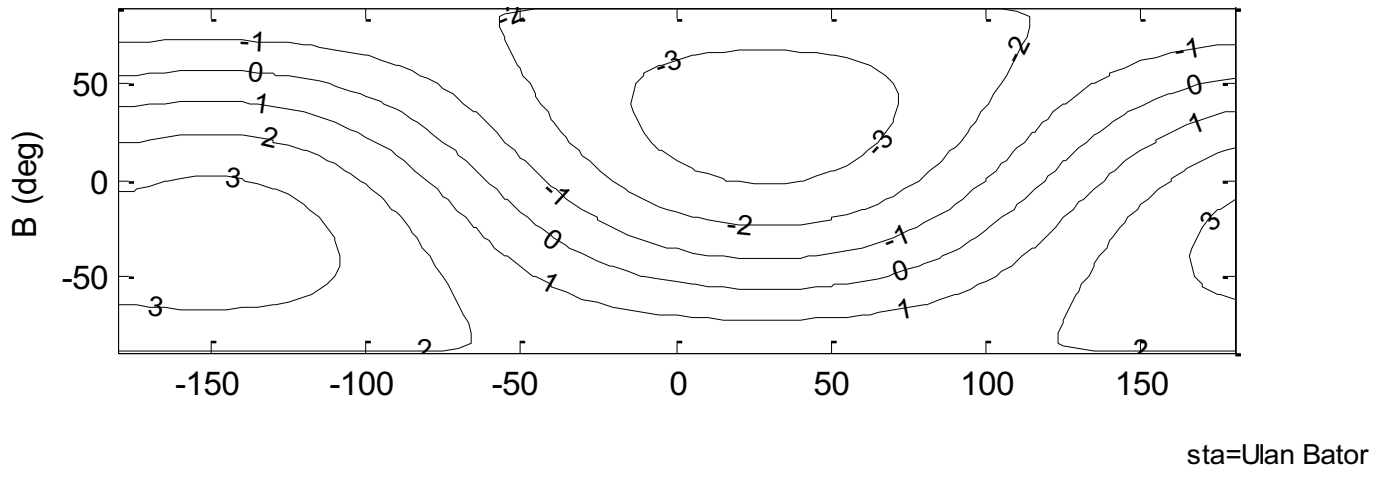

(c) North error (m)

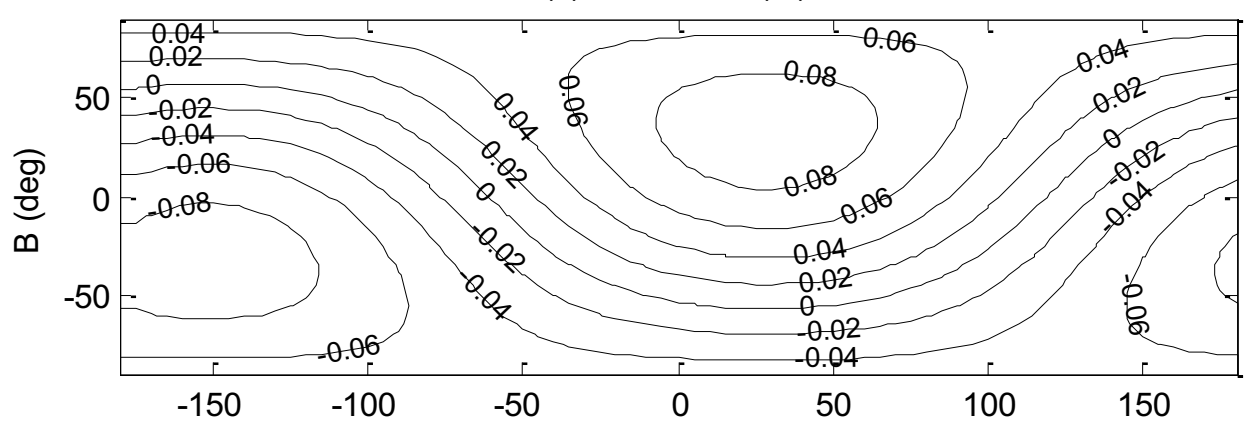

(d) Up error (m)

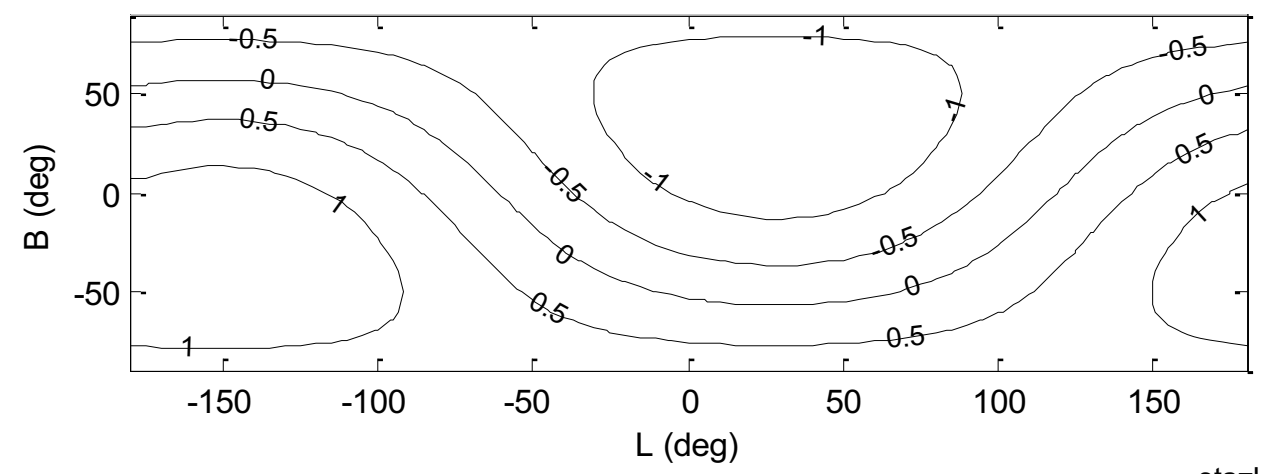

sta=Ulan Bator

Fig. 4 Error vector angle and positioning error distributions at Ulan Bator 
(a) Angles between LOS and $r_{e}$ (deg)

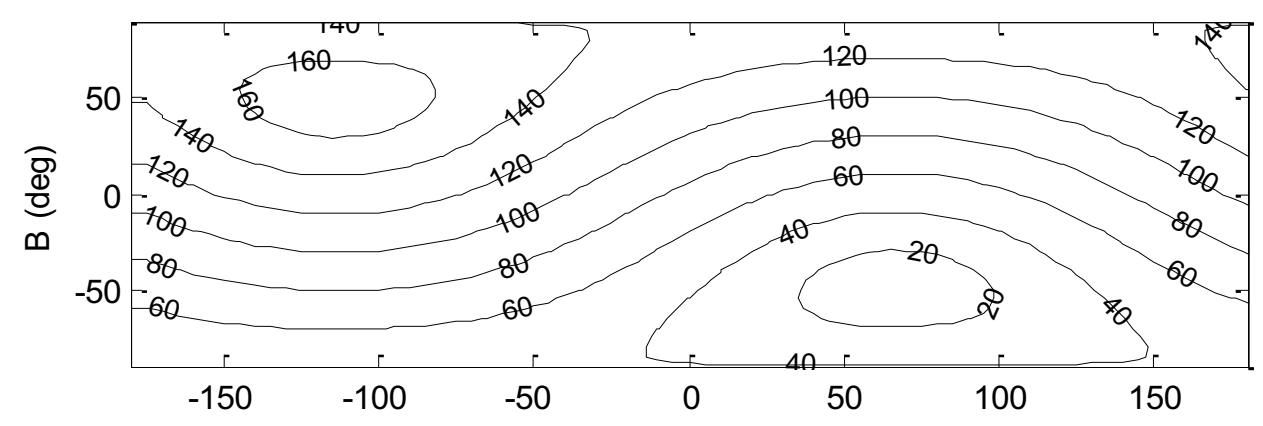

(b) East error (m)

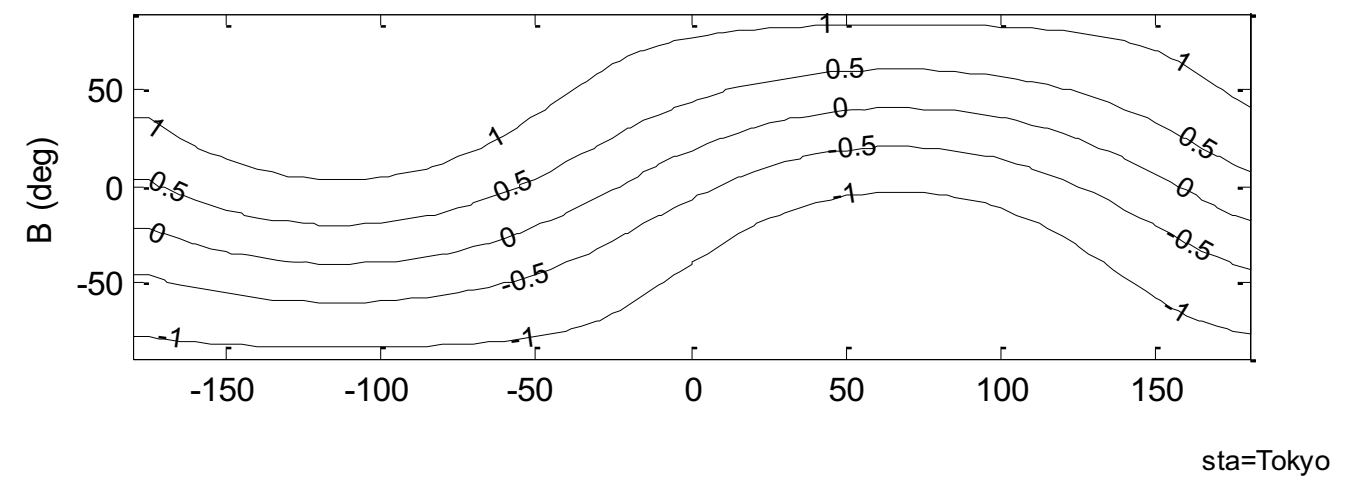

(c) North error (m)

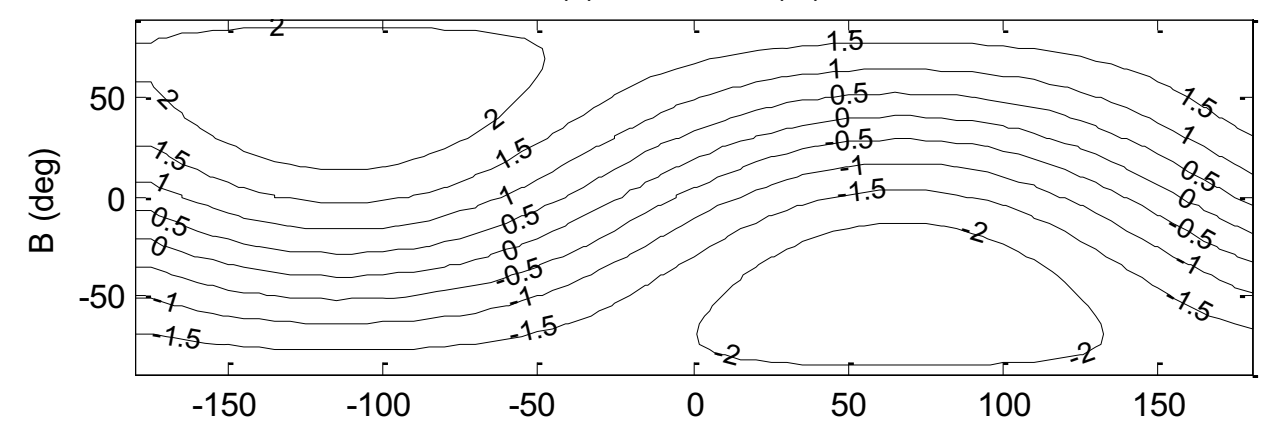

(d) Up error (m)

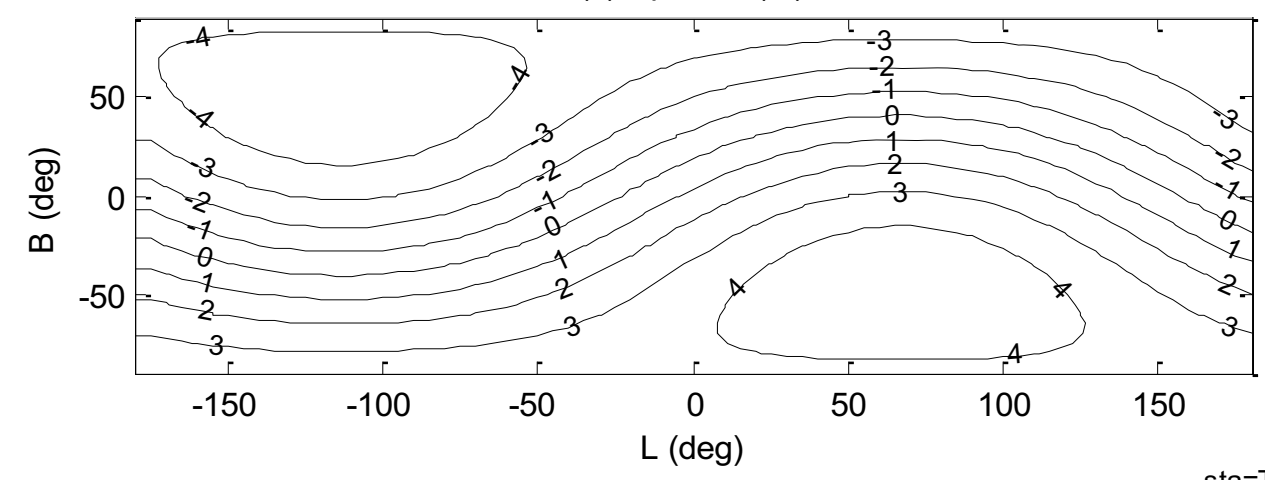

Fig. 5 Error vector angle and positioning error distributions at Tokyo 
(a) Angles between LOS and $r_{e}$ (deg)

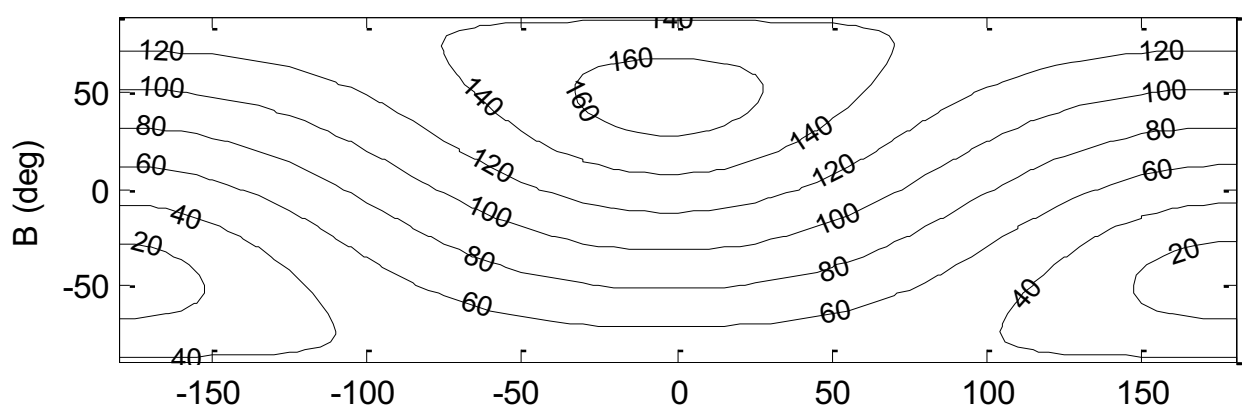

(b) East error (m)

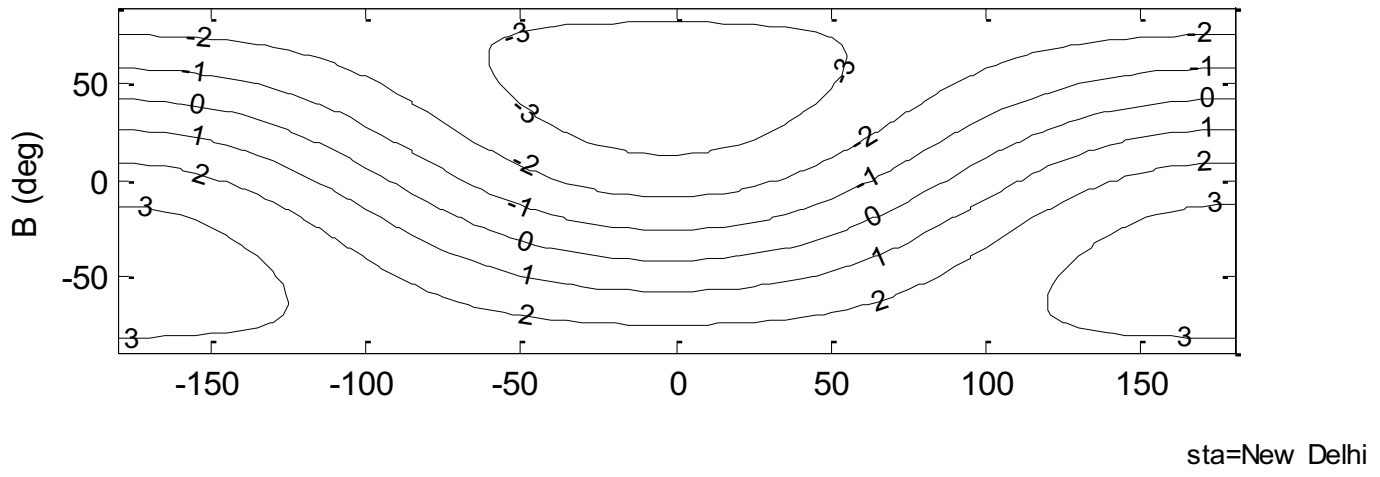

(c) North error (m)

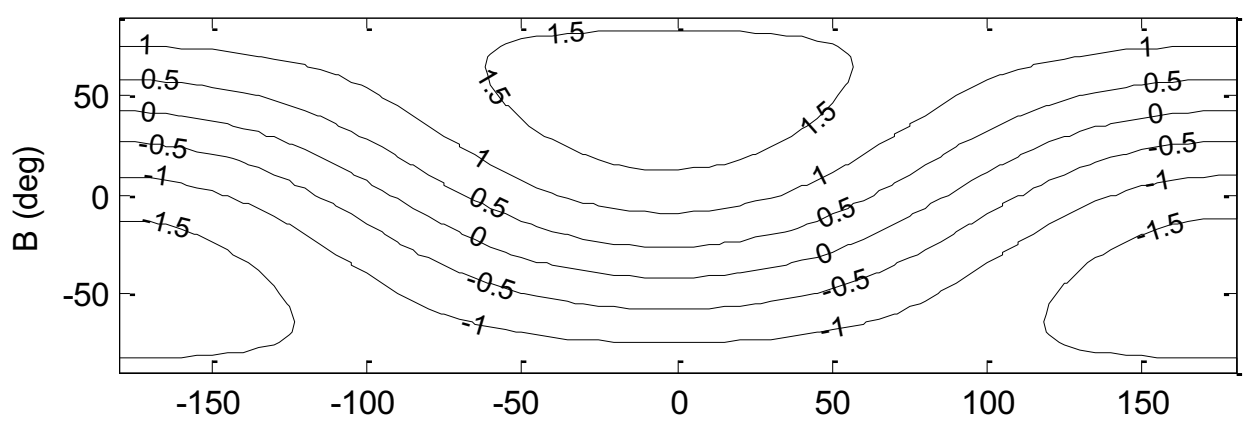

(d) Up error (m)

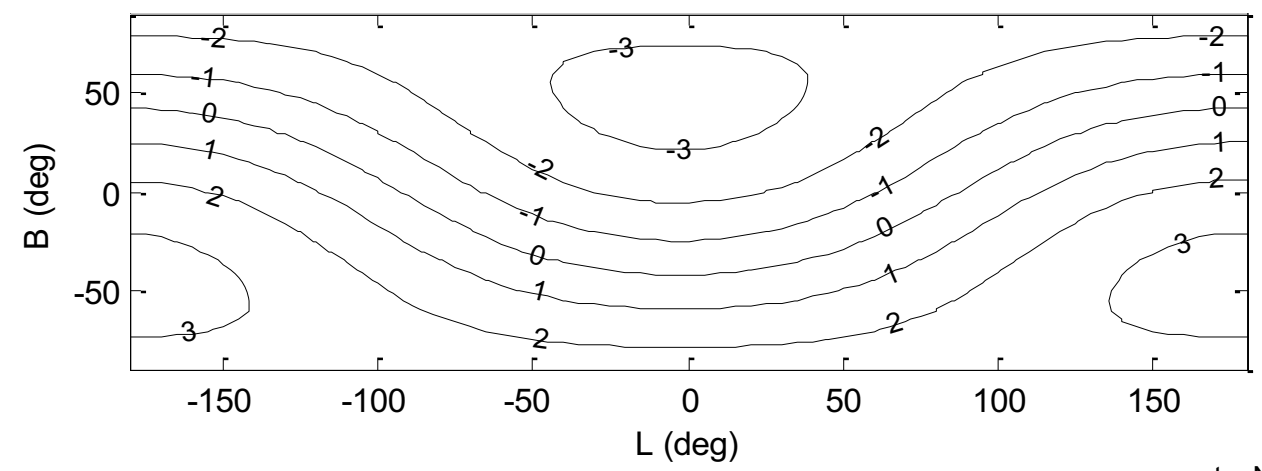

Fig. 6 Error vector angle and positioning error distributions at New Delhi 
(a) Angles between LOS and $r_{e}$ (deg)

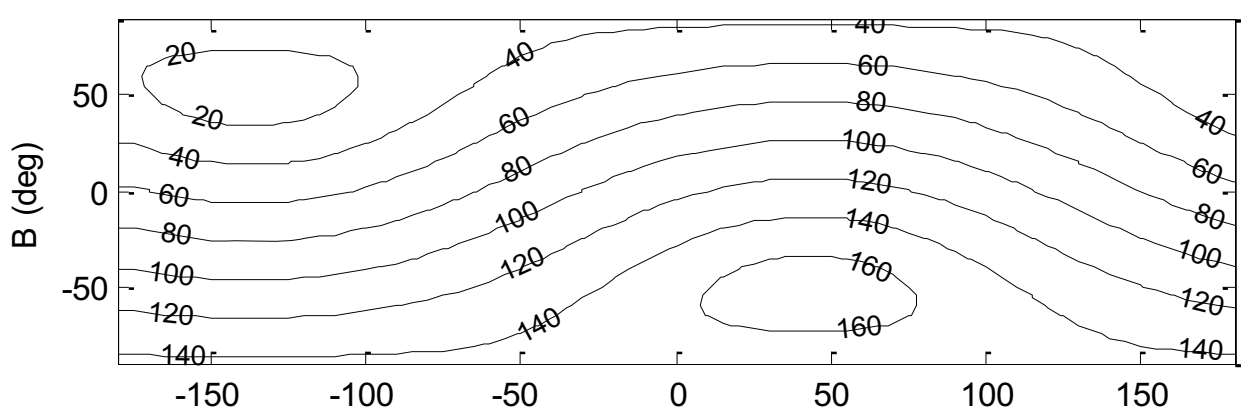

(b) East error (m)

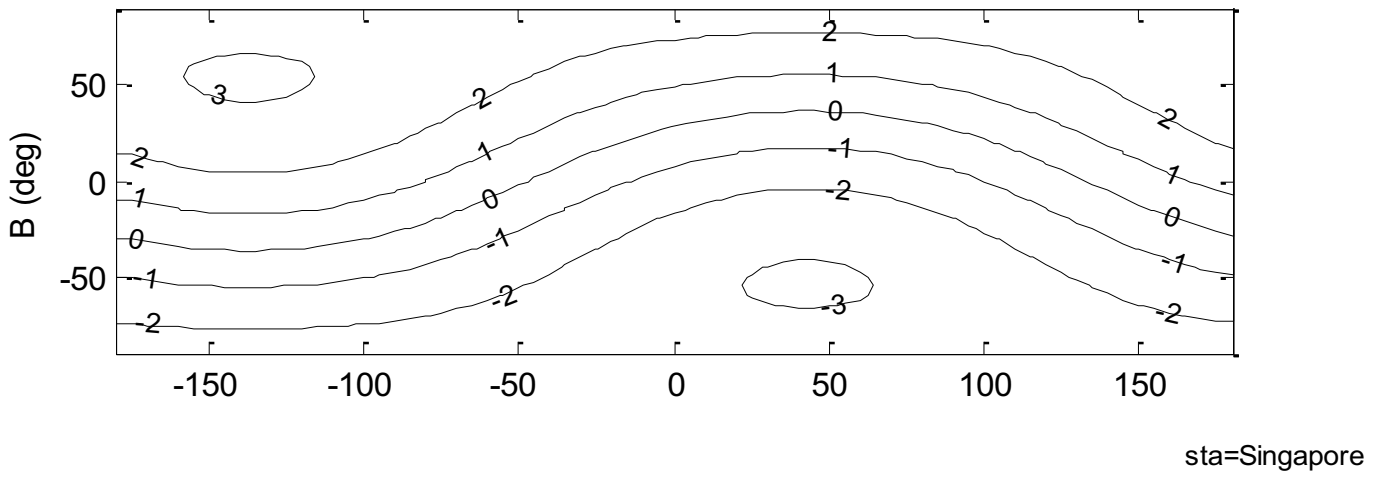

(c) North error (m)

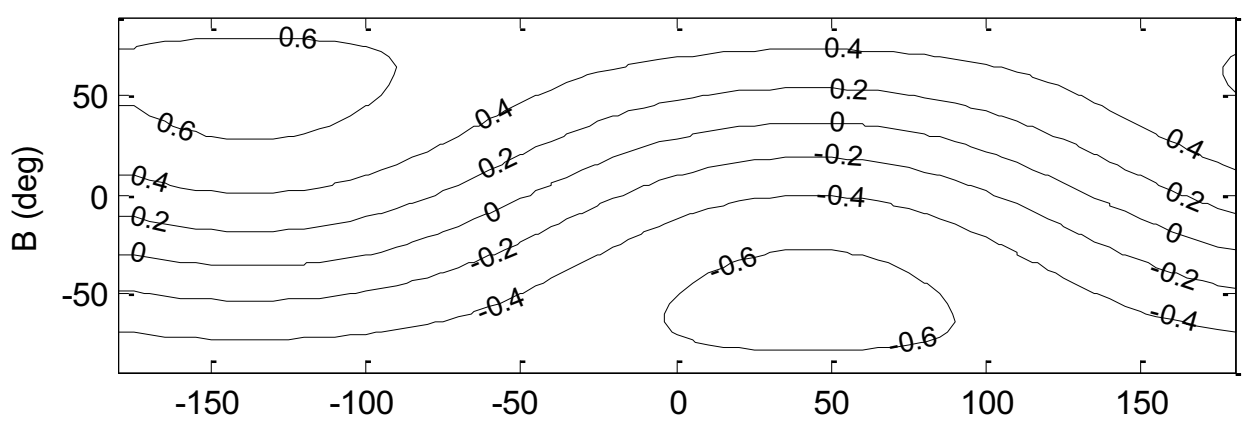

(d) Up error (m)

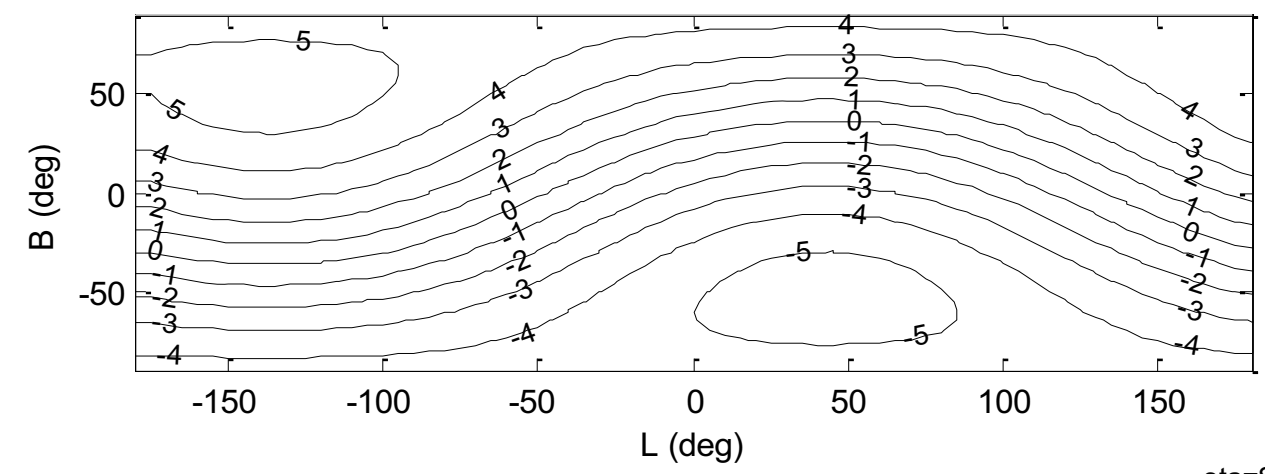

Fig. 7 Error vector angle and positioning error distributions at Singapore 
(a) Angles between LOS and $r_{e}$ (deg)

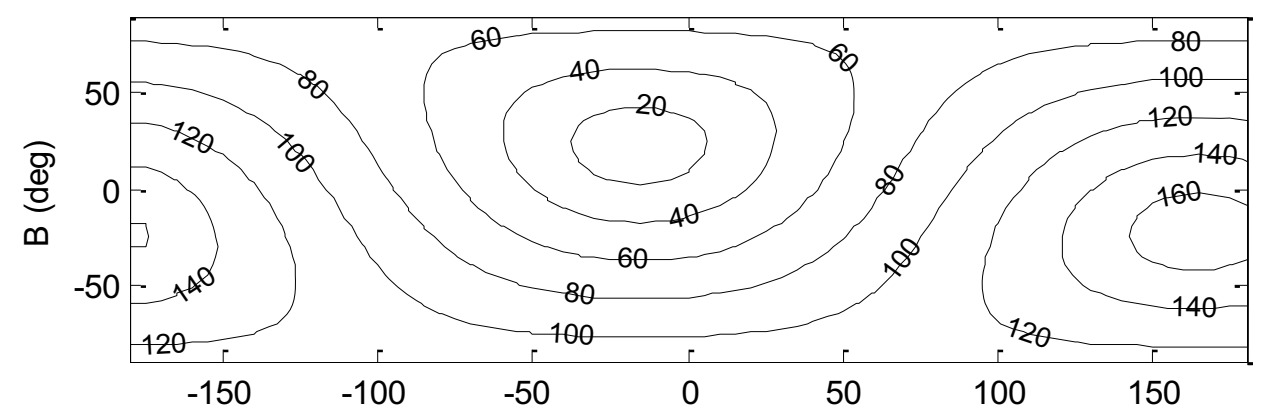

(b) East error (m)

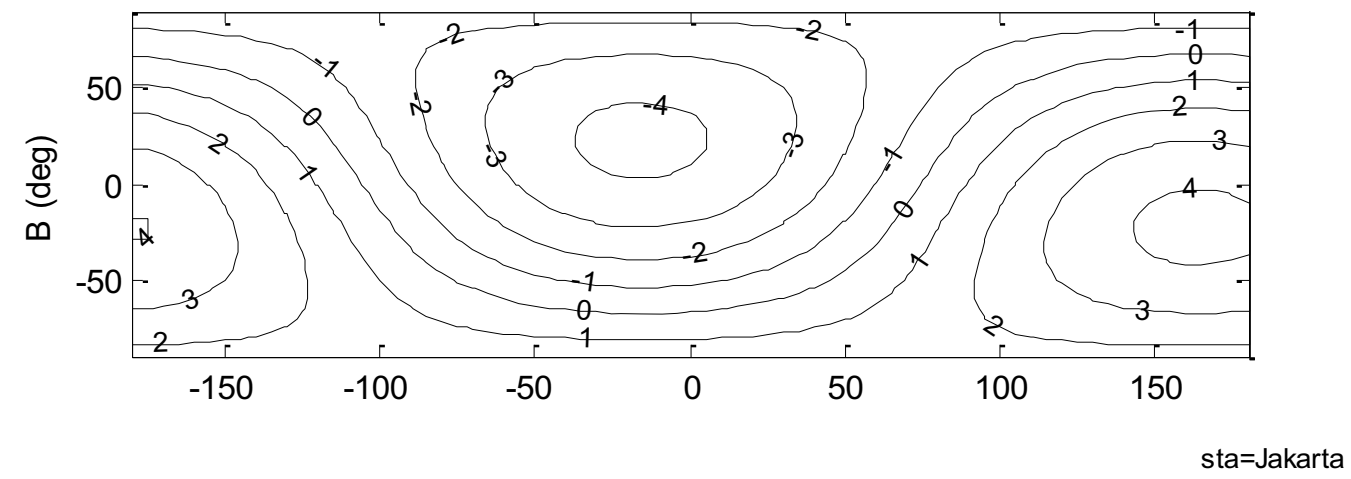

(c) North error (m)

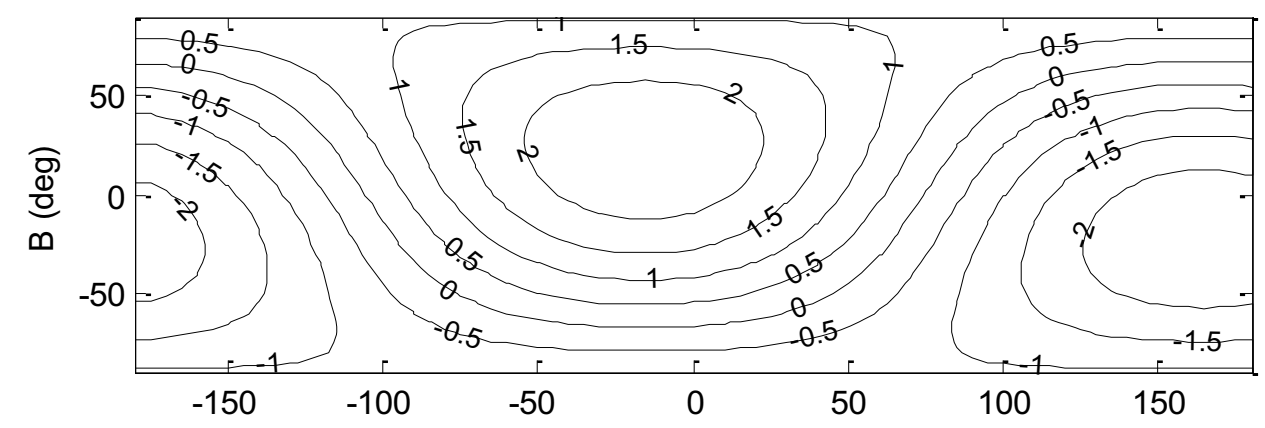

(d) Up error (m)

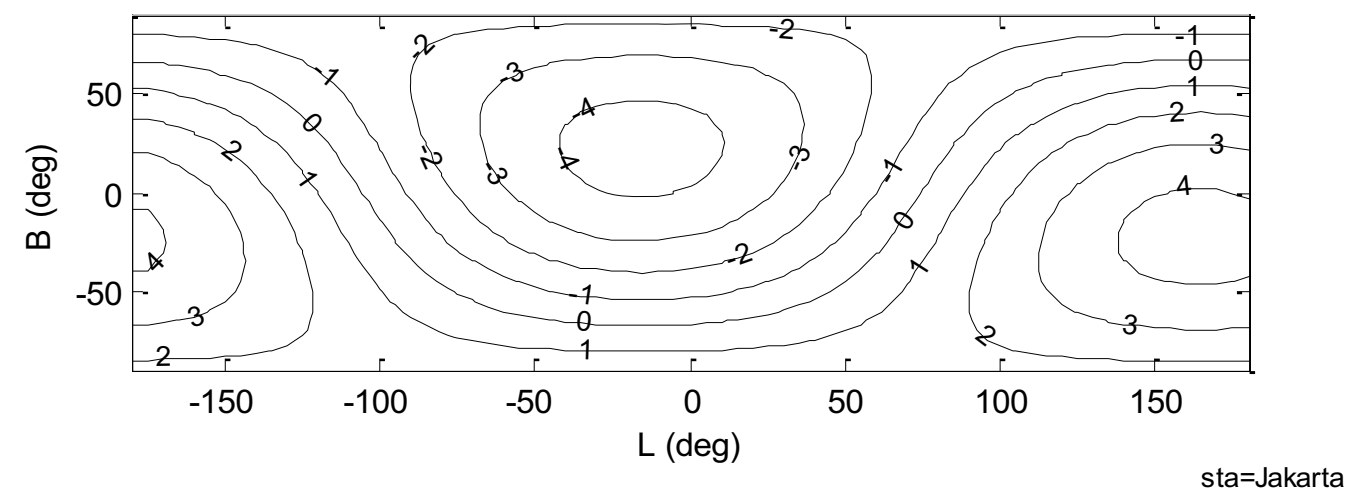

Fig. 8 Error vector angle and positioning error distributions at Jakarta 
(a) Angles between LOS and $r_{e}$ (deg)

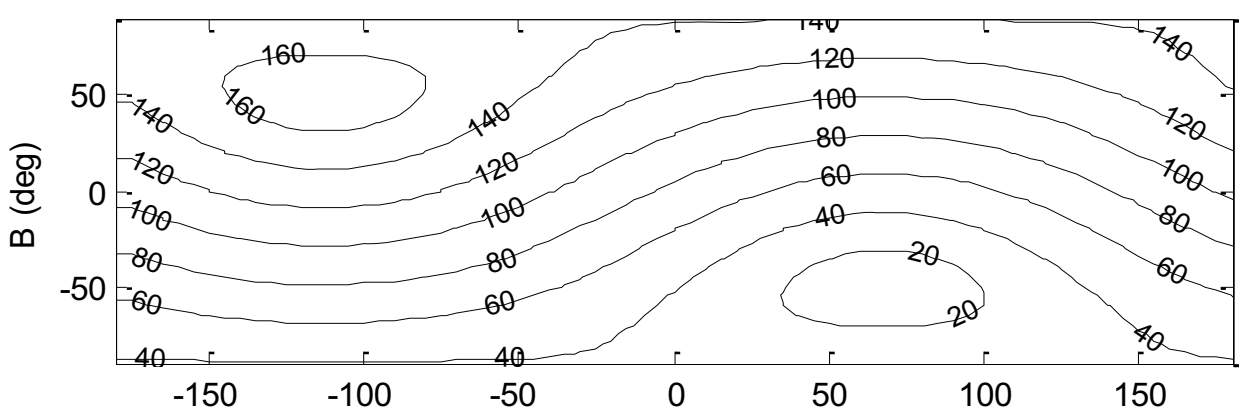

(b) East error (m)

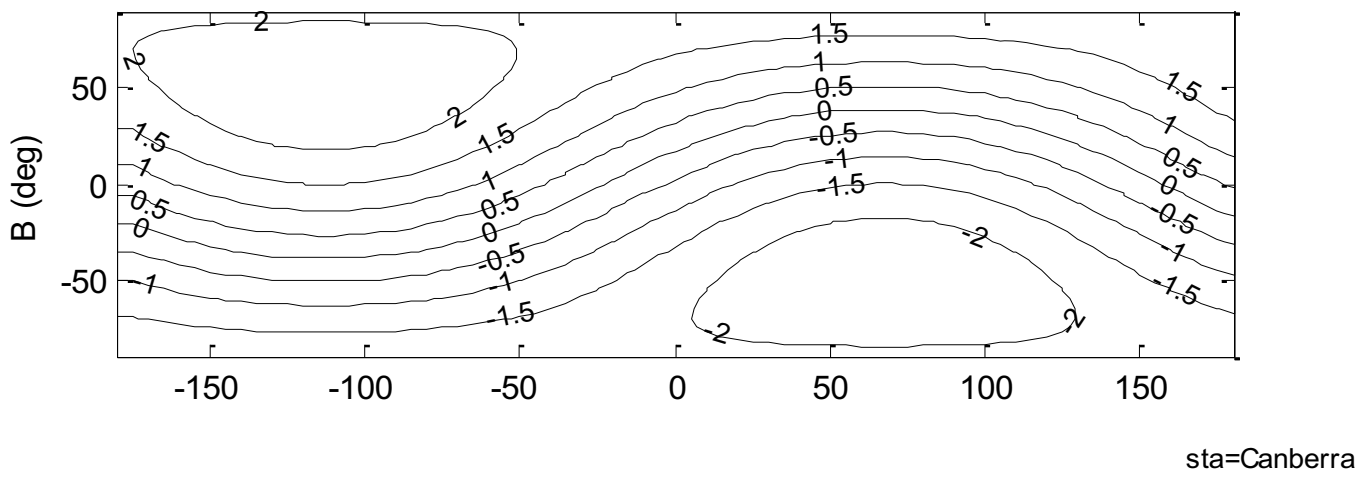

(c) North error (m)

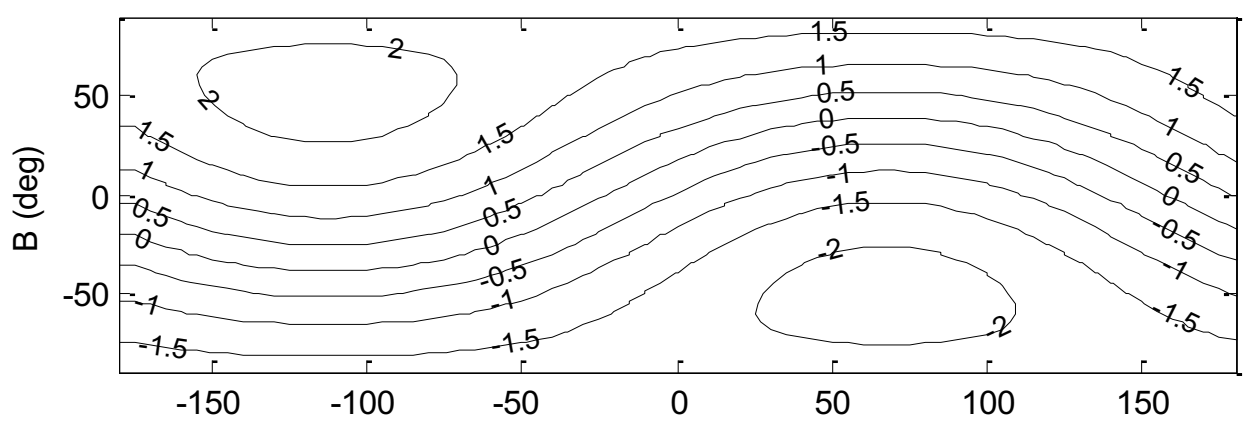

(d) Up error (m)

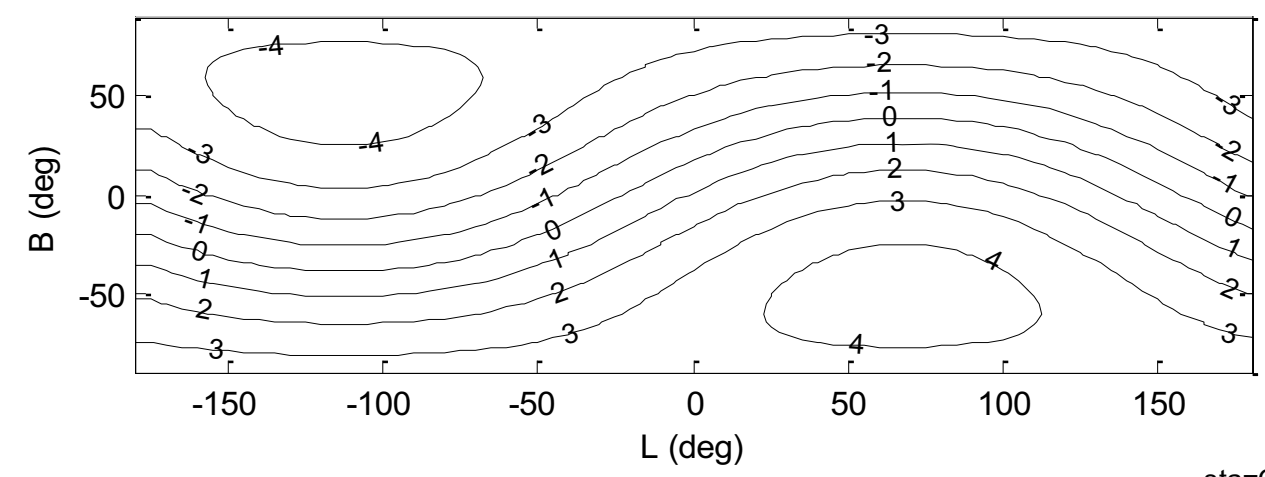

sta=Canberra

Fig. 9 Error vector angle and positioning error distributions at Canberra 
(a) Angles between LOS and $r_{e}$ (deg)

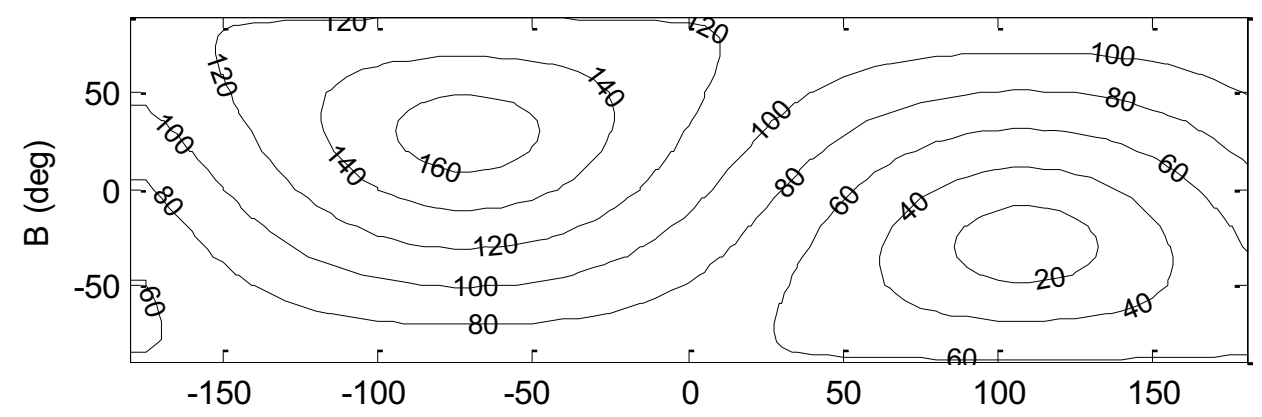

(b) East error (m)

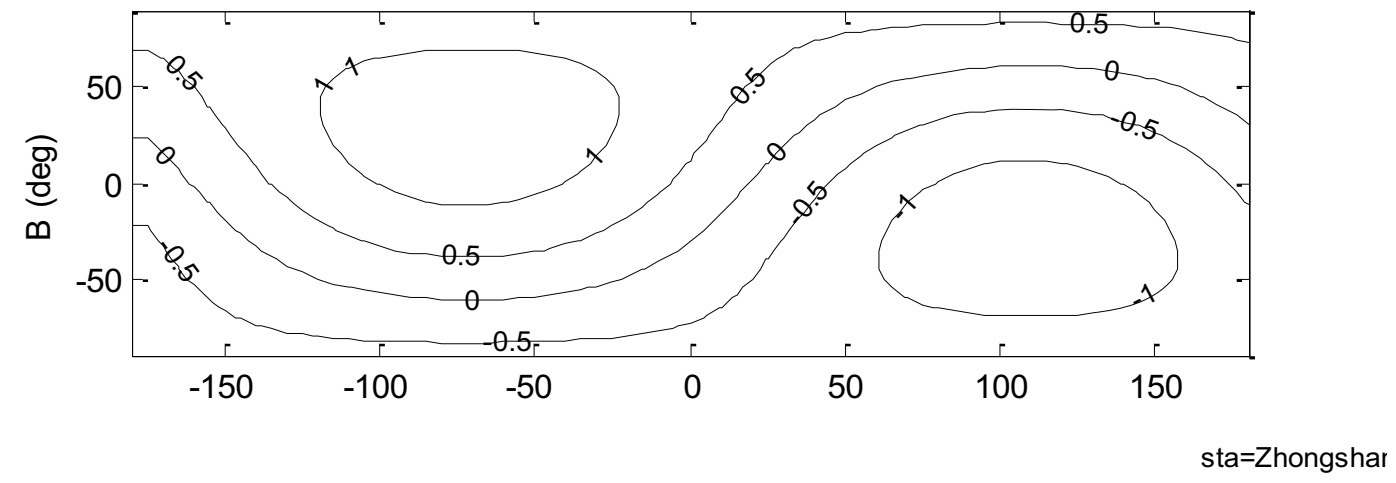

(c) North error (m)

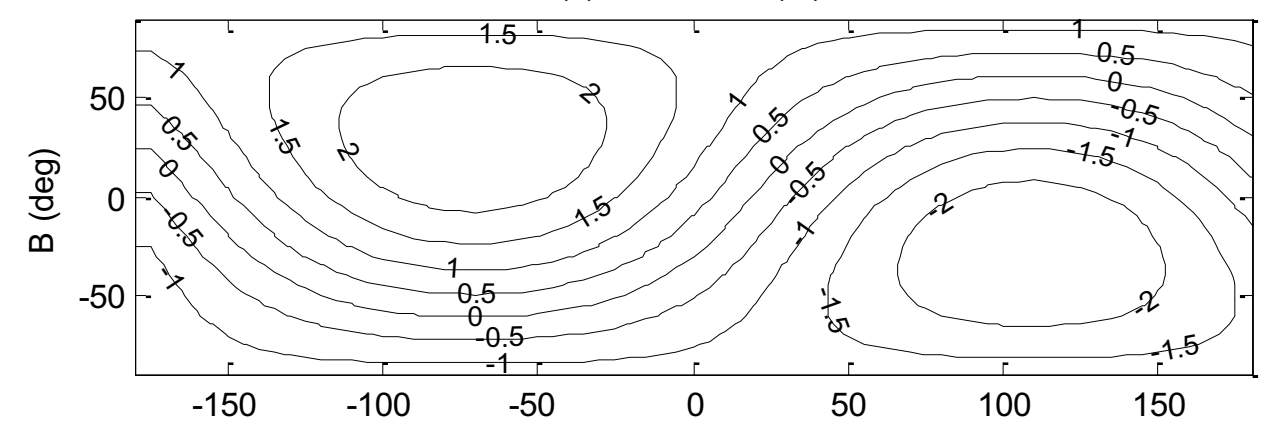

(d) Up error (m)

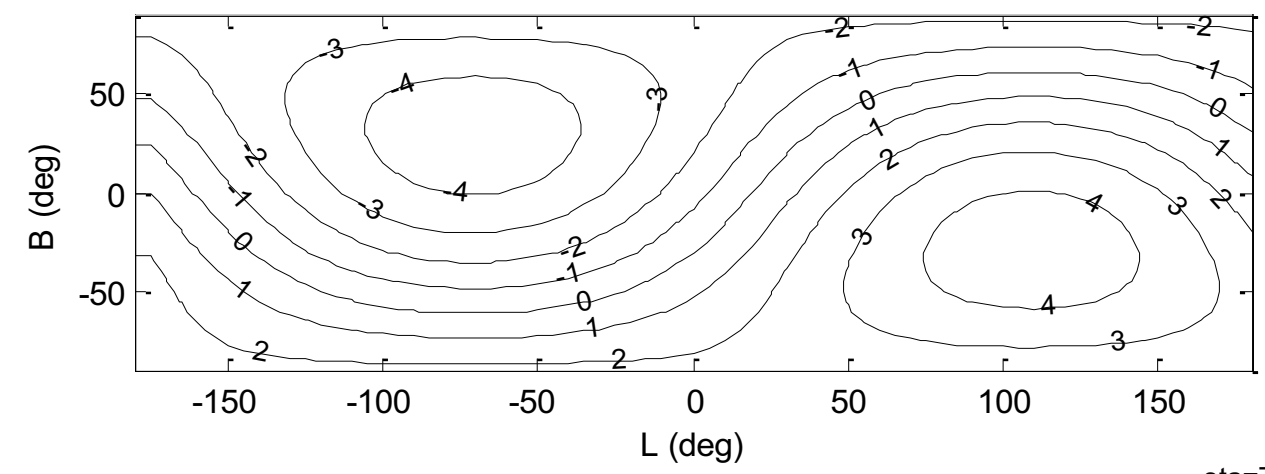

Fig. 10 Error vector angle and positioning error distributions at Zhongshan 
It can be noted that the positioning error caused by ephemeris error along the LOS is much more bigger than that perpendicular to the LOS. Since introduced ephemeris error is added to different satellites in each station, the caused positioning error is distinct from each station. Even for the same satellite, the positioning error in different stations is different. In Beijing and New Delhi, the same satellite is used to added ephemeris error. The positioning error in north and east directions is much more bigger than that in upper direction in Beijing station. While the positioning error in east and upper directions is much more bigger than that in north direction in New Delhi station.

It is obvious that different orientation of ephemeris error vector takes place at different station. Meanwhile the influence of ephemeris error is remarkable when the ephemeris error is along the LOS direction where positioning error in east, north and up directions has the most value (maximum or minimum). Except from Singapore station, the maximum and the minimum values interspersed appear in the three directions under the same angle condition. To clearly display the results, the statistics data of accuracy error is listed in the Table 2.

Table 2. Maximum positioning error statistics from observation stations.

\begin{tabular}{|l|c|c|c|c|c|c|}
\hline Station & East error (m) & North error (m) & Up error (m) & 3D error (m) & PDOP & SVID \\
\hline Beijing & 4.49 & 4.87 & 1.09 & 6.72 & 2.75 & 29 \\
\hline Ulan Bator & 3.66 & 0.09 & 1.44 & 3.93 & 2.10 & 25 \\
\hline Tokyo & 1.45 & 2.48 & 4.83 & 5.62 & 2.47 & 23 \\
\hline New Delhi & 3.64 & 1.84 & 3.35 & 5.28 & 3.52 & 29 \\
\hline Singapore & 3.07 & 0.67 & 5.45 & 6.29 & 2.50 & 27 \\
\hline Jakarta & 4.25 & 2.44 & 4.39 & 6.58 & 2.91 & 17 \\
\hline Canberra & 2.40 & 2.20 & 4.46 & 5.52 & 2.45 & 23 \\
\hline Zhongshan & 1.32 & 2.50 & 4.62 & 5.41 & 2.59 & 30 \\
\hline
\end{tabular}

The minimum value of three dimensional (3D) error is close to zero, not exceeding $1 \mathrm{~cm}$. Here the PDOP value, as an indicator of 3D positioning accuracy, is calculated. The maximum PDOP value is less than 3.52. The last column in the Table 2 gives Satellite-Vehicle Identification Number (SVID). From the Table 2 the maximum 3D positioning error ranges from 3.93 meters to 6.72 meters, while positioning errors exceed 5 meters at most of stations.

\section{CONCLUSIONS AND DISCUSSIONS}

In this article with simulation observations from Beijing, Ulan Bator, Tokyo, New Delhi, Singapore, Jakarta, Canberra and Zhongshan stations, results of simulation analysis show that the ephemeris error influences on GPS single point positioning accuracy to a large extent. Given ephemeris error existing in one satellite, magnitude of maximum positioning error approaches that of ephemeris error. Furthermore the maximum 3D positioning error ranges from 3.93 meters to 6.72 meters considering 5 meters ephemeris error. The influence will become more complicated when ephemeris error taking place in more satellites. Meanwhile, when the ephemeris error is along the LOS direction, the influence is remarkable. The minimum positioning error exists in direction perpendicular to LOS direction.

According the above results, the maximum position error must exist in ephemeris error being along the LOS direction in every satellite if ephemeris error vector is added in two and more GPS satellites. In this work the complicated analysis don't carried out for numerous 
variables are introduced. Considering that the surface of error sphere doesn't possess random characteristics, the relationship between the position error and range measurement error can't be satisfied with the applicable condition of Equation (6). Though the GPS satellite clock bias, drift and drift-rate are determined and these changes are available to all GPS users as clock error coefficients broadcast in the navigation message, the residual error of model satellite clock can be converted to ephemeris error along the LOS direction and affect position estimate. The focal point in this work is the position error distribution when ephemeris error locates on the surface of error sphere. The detailed analysis of combined effect for satellite ephemeris and clock error will be included in an extended study in future.

Acknowledgements. This study is supported by the National Basic Research and Development Program of China (Grant No. 2007CB815501), the Key Research Program of the Chinese Academy of Sciences (Grant No. KJCX2-EW-J01) and the Knowledge Innovation Program of the Chinese Academy of Sciences (Grant No. KGCX2-EW-407-1).

\section{REFERENCES}

George W., and Collins II. (2004) The Foundations of Celestial Mechanics. Case Western Reserve University.

Kaplan E., and Hegarty C. (2006) Understanding GPS: Principles and Applications. Artech House.

Langley R. (1999) Dilution of precision. GPS World, Vol. 10, No. 5, 52-59.

Misra P., and Enge P. (2006) Global Positioning System: Signals, Measurements, and Performance. Ganga-Jamuna Press.

Warren D., and Raquet J. (2002) Broadcast vs Precise GPS Ephemerides: A Historical Perspective, Proceedings of the 2002 National Technical Meeting of The Institute of Navigation, San Diego, CA, January 2002, p. 733-741.

Received: 2012-10-08,

Reviewed: 2013-02-18, by Z. Rzepecka, and 2013-04-26,

Accepted: 2013-06-13. 